\title{
SYMMETRIC POLYNOMIALS WITH NON-NEGATIVE COEFFICIENTS
}

\section{KUIPERS AND B. MEULENBELD}

1. Introduction. Brunn [1] proved a theorem on a determinant (an alternant) the elements of which are elementary symmetric functions of positive variables. This theorem reflected by us in a sharper form and applied to polynomials (Theorem 1) is the basis of further investigation. A special case is Theorem 2 , important applications of which are Theorems 3 and 4 . In $\$ 2$ we prove these results and in $\S 3$ we give some examples. In $\$ 4$ the foregoing is applied to absolutely monotonic functions; the result is Theorem 5, a generalization of a theorem of Rosenbloom [2]. In $\$ 5$ an extension of Theorem 3 is deduced (Theorem 6) by considering a function of two variables.

2. Let $S_{j}$ be the elementary symmetric function of $n$ variables $x_{1}, x_{2}, \cdots, x_{n}$, defined by

$$
\begin{array}{lr}
S_{j}=\sum x_{1} x_{2} \cdots x_{i} & \text { for } j=1,2, \cdots, n ; \\
S_{0}=1 ; S_{j}=0 & \text { for } j=-1,-2, \cdots
\end{array}
$$

Theorem 1. The determinant $\left(S_{k_{i j}}\right), i=1,2, \cdots, q ; j=1,2, \cdots$, $q$, with

$$
\begin{aligned}
k_{i, m}-k_{i, m+1} & =k(m)>0, \\
k_{m+1, i}-k_{m, i} & =k^{*}(m)>0, \\
i & =1,2, \cdots, q ; m=1,2, \cdots, q-1,
\end{aligned}
$$

can be written as a symmetric polynomial in $x_{1}, x_{2}, \cdots, x_{n}$ with nonnegative coefficients.

Proof. Obviously the determinant in question is a symmetric polynomial in the considered variables.

For $n=1$ the determinant equals zero or unity or a power of $x_{1}$. Now applying induction we assume the assertion to be true for $n-1$ and prove the truth for the case $n$. Therefore we put

$$
S_{k}=S_{k}^{\prime}+x_{n} S_{k-1}^{\prime},
$$

where $S_{k}^{\prime}$ differs from $S_{k}$ in referring to the variables with $x_{n}$ left out. Substituting (2) in the determinant we can expand this in increasing powers of $x_{n}$, hence

Received by the editors May 12, 1952 and, in revised form, April 4, 1954. 


$$
\left(S_{k_{i j}}\right)=\sum_{k=0}^{q} A_{k} x_{n}^{k}
$$

where $A_{k}$ is a sum of determinants the elements of which are elementary symmetric functions of $x_{1}, x_{2}, \cdots, x_{n-1}$. The element indices satisfy (1), hence from our assumption it follows that each $A_{k}$ is a polynomial in $x_{1}, x_{2}, \cdots, x_{n-1}$ with non-negative coefficients. Because of (3) our assertion is proved.

An immediate consequence of Theorem 1 is

Theorem 2. Let $\sigma_{j}=(-1)^{i} S_{j}$. Then the determinant $\left(\sigma_{m+i-k_{j}}\right)\left(i=0,1, \cdots, q ; j=0,1, \cdots, q ; 0=k_{0}<k_{1}<\cdots<k_{q}\right)$ multiplied by the factor $(-1)^{M}$, where

$$
\begin{aligned}
M= & m+\left(m-k_{1}\right)+\left(m-k_{2}\right)+\cdots \\
& +\left(m-k_{q}\right)+1+2+\cdots+q,
\end{aligned}
$$

is expressible as a symmetric polynomial in $x_{1}, x_{2}, \cdots, x_{n}$ with nonnegative coefficients.

Now we prove the following

THEOREM 3. Let

$$
f_{h+1}(x) \equiv a_{h 0}+a_{h 1} x+\cdots+a_{h, n+p} x^{n+p} \quad(h=0,1, \cdots, n-1),
$$

where $n \geqq 2$ and $p \geqq 0$, be $n$ polynomials with real coefficients such that all determinants $D$ of the nth order, taken from the matrix $\left|a_{i j}\right|, i=0,1$, $\cdots, n-1 ; j=0,1, \cdots, n+p$, are non-negative.

If for $n$ variables $x_{1}, x_{2}, \cdots, x_{n}$, with $x_{i} \neq x_{j}$ for $i \neq j$, we put $V$ $=V(x)=$ the determinant $\left(x_{i}^{j}\right), i=1,2, \cdots, n ; j=0,1, \cdots, n-1$, then the expression

$$
\left(f_{i}\left(x_{j}\right)\right) / V \text {. }
$$

can be written as a symmetric polynomial in $x_{1}, x_{2}, \cdots, x_{n}$ with nonnegative coefficients.

Proof. From a theorem of Garbieri [3] it follows that (4) is equal to the determinant of $(n+p+1)$ th order

$$
\begin{aligned}
& \left(B_{i j}\right), B_{i j}=a_{i j} \quad(i=0,1, \cdots, n-1 ; j=0,1, \cdots, n+p), \\
& B_{i j}=\sigma_{i-j} \quad(i=n, n+1, \cdots, n+p ; j=0,1, \cdots, n+p),
\end{aligned}
$$

where $\sigma_{j}$ is defined in Theorem 2. By expanding (5) in terms of the $(p+1)$-line minors of the last $p+1$ rows (let $i$ denote the rows) we see that $\left(B_{i j}\right)$ is the sum of a number of expressions each of which is 
a product of a determinant $D$, the corresponding determinant $\left(\sigma_{n+i-k_{j}}\right), i=1,2, \cdots, p+1 ; j=1,2, \cdots, p+1 ; 1 \leqq k_{1}<k_{2}<\cdots$ $<k_{p+1} \leqq n+p+1$, and the factor $(-1)^{s}$ where $S=(n+1)+(n+2)$ $+\cdots+(n+p+1)+k_{1}+k_{2}+\cdots+k_{p+1}$. Now the exponent $M$ (see Theorem 2) related to this last determinant is equal to $\left(n+1-k_{1}\right)$ $+\cdots+\left(n+1-k_{p+1}\right)+1+2+\cdots+p$, so that $M \equiv S(\bmod 2)$. From this conclusion and Theorem 2 the assertion follows.

A special case of Theorem 3 (put $f_{h}(x)=x^{k_{n}}, h=1,2, \cdots, n$ ) is

Theorem 4 (P. C. Rosenbloom [2, p. 459]). If $k_{1}, k_{2}, \cdots, k_{n}$ are integers with $0 \leqq k_{1}<k_{2}<\cdots<k_{n}$, then

$$
\left(x_{i}^{k_{j}}\right) / V ; \quad i, j=1,2, \cdots, n,
$$

is a symmetric polynomial in $x_{1}, x_{2}, \cdots, x_{n}$ with non-negative coeffcients.

REMARK. The last result can also be obtained by application of a theorem of $\mathrm{H}$. Naegelsbach [4].

Acting in this way we find for the expression (6) the determinant

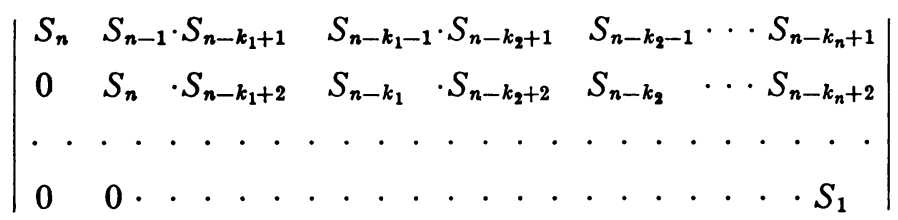

whose elements satisfy the conditions of Theorem 1 .

3. Examples. 1. If for $h=1,2, \cdots, n ; p \geqq 0$,

$$
f_{h}(x)=\sum_{i=0}^{n+p} a_{h}^{k+i-1} x^{i} \quad \text { with } 0<a_{1}<\cdots<a_{n},
$$

then $\left(f_{i}\left(x_{j}\right)\right) / V ; i, j=1,2, \cdots, n$, is a symmetric polynomial in $x_{1}, x_{2}, \cdots, x_{n}$ with non-negative coefficients.

This follows from the fact that the determinants of the $n$th order taken from the matrix

$$
\left|a_{i}^{i+j-2}\right|, \quad i=1,2, \cdots, n ; j=1,2, \cdots, n+p+1,
$$

divided by the positive number $V(a)$ are polynomials in the positive $a_{1}, a_{2}, \cdots, a_{n}$ with non-negative coefficients, as follows from Theorem 4.

2. If the determinants of the nth order taken from the matrix $\left|a_{i j}\right|$, $i=1,2, \cdots, n ; j=1,2, \cdots, n+p$, are positive, and if 


$$
A_{i j}=\sum_{q=1}^{n+p} \sum_{h=1}^{n} a_{i q} x_{h}^{q} x_{j}^{k_{h}}, \quad i, j=1,2, \cdots, n,
$$

where the $k_{h}$ are integers with $0 \leqq k_{1}<\cdots<k_{n}$, then $\left(A_{i j}\right) / V^{2}(i, j$ $=1,2, \cdots, n)$ is a symmetric polynomial with non-negative coefficients.

Proof. Putting $f_{i}(x) \equiv \sum_{q=1}^{n+p} a_{i q} x^{q}(i=1,2, \cdots, n)$, we have

$$
A_{i j}=\sum_{h=1}^{n} x_{i}^{k_{k}} \sum_{q=1}^{n+p} a_{i q} x_{h}^{q}=\sum_{h=1}^{n} x_{i}^{k_{h}} f_{i}\left(x_{h}\right) \text {, }
$$

so that

$$
\left(A_{i j}\right)=\left(x_{i}^{k_{j}}\right)\left(f_{i}\left(x_{i}\right)\right) \quad(i, j=1,2, \cdots, n) .
$$

Application of Theorem 3 completes the proof.

4. THEOREM 5. If $f(x)$ and $g(x)$ are power series with non-negative coefficients converging in the interval $0 \leqq x<a$, then the expression

$$
\begin{array}{r}
(-1)^{n-1} \operatorname{det}\left|1 x_{i}^{1} \cdots x_{i}^{n-3} f\left(u x_{i}\right) g\left(v x_{1} \cdots x_{i-1} x_{i+1} \cdots x_{n}\right)\right| / V, \\
i=1,2, \cdots, n,
\end{array}
$$

can be written as a power series in the variables $x_{1}, x_{2}, \cdots, x_{n}, u, v$ with non-negative coefficients converging in the range

$$
0 \leqq x_{1}, x_{2}, \cdots, x_{n}<a ; 0 \leqq u \leqq 1 ; 0 \leqq v \leqq \frac{1}{a^{n-1}} .
$$

Proof. Putting

$$
f(x)=\sum_{q=0}^{\infty} a_{q} x^{q}, \quad g(x)=\sum_{m=0}^{\infty} b_{m} x^{m}, \quad a_{q} \geqq 0, b_{m} \geqq 0,
$$

then (7) can be expressed as

$$
\begin{aligned}
& (-1)^{n-1} \sum_{q=0}^{\infty} a_{q} u^{q}\left|1 x_{i}^{1} \cdots x_{i}^{n-3} x_{i}^{q} g\left(v x_{1} \cdots x_{i-1} x_{i+1} \cdots x_{n}\right)\right| / V \\
& =(-1)^{n-1} \sum_{q=0}^{\infty} \sum_{m=0}^{\infty} a_{q} b_{m} u^{q} v^{m}\left|1 x_{i}^{1} \cdots x_{i}^{n-8} x_{i}^{q}\left(x_{1} \cdots x_{i-1} x_{i+1} \cdots x_{n}\right)^{m}\right| / V \\
& =(-1)^{n-1} \sum_{q=0}^{\infty} \sum_{m=0}^{\infty} a_{q} b_{m} u^{q} v^{m}\left|x_{i}^{m} x_{i}^{m+1} \cdots x_{i}^{m+n-3} x_{i}^{m+q} 1\right| / V \\
& =\sum_{q=n-2}^{\infty} \sum_{m=0}^{\infty} a_{q} b_{m} u^{q} v^{m}\left|1 x_{i}^{m} x_{i}^{m+1} \cdots x_{i}^{m+n-8} x_{i}^{m+q}\right| / V,
\end{aligned}
$$


so that from Theorem 4 the correctness of this theorem follows.

REMARK. For $n=3$ this theorem is a result of Rosenbloom about absolutely monotonic functions [2]. (The range of $u$ and $v$ given there is not quite correct.)

5. Theorem 6. Let $F(x, y)$ be the function $\sum_{i=0}^{n+p} \sum_{j=0}^{n+p} a_{i j} x^{i} y^{j}$ $(p \geqq 0)$, with the property that all determinants $D$ of the nth order taken from the matrix $\left|a_{i j}\right|$ are non-negative. Let $\left(x_{1}, x_{2}, \cdots, x_{n}\right)$ and $\left(y_{1}, y_{2}, \cdots, y_{n}\right)$ with $x_{i} \neq x_{j}, y_{i} \neq y_{j}(i \neq j)$ be two sets of variables.

Then the expression

$$
\left(F\left(x_{i}, y_{j}\right)\right) / V(x) V(y) .
$$

is a polynomial, symmetric in $x_{1}, x_{2}, \cdots, x_{n}$ as well as in $y_{1}, y_{2}, \cdots$, $y_{n}$ with non-negative coefficients.

Proof. On account of another theorem of Garbieri [3] the expression (8) is identical with

$$
(-1)^{p-1}\left(t_{i j}\right)
$$$$
i, j=0,1, \cdots, n+2 p+1,
$$

where

$$
\begin{aligned}
& t_{i j}=a_{i j} \\
& (i, j=0,1, \cdots, n+p) \\
& =\sigma_{j-i-p-1}^{\prime} \\
& (i=0,1, \cdots, n+p, j=n+p+1, \cdots, n+2 p+1) \\
& =\sigma_{i-j-p-1} \quad(i=n+p+1, \cdots, n+2 p+1, j=0,1, \cdots, n+2 p+1) \text {, }
\end{aligned}
$$

where $(-1)^{i} \sigma_{j}$ and $(-1)^{i} \sigma_{j}^{\prime}$ are the elementary symmetric functions of $x_{1}, x_{2}, \cdots, x_{n}$ and $y_{1}, y_{2}, \cdots, y_{n}$ respectively. We develop the determinant in (9) in terms of the $(p+1)$-line minors of the last $p+1$ rows. The term corresponding with the minor indicated by the column-indices $k_{1}, k_{2}, \cdots, k_{p+1}$, say $\mathfrak{M}$, possesses the sign

$$
(-1)^{(n+p+2)+(n+p+3)+\cdots+(n+2 p+2)+k_{1}+k_{2}+\cdots+k_{p+1}}=(-1)^{M} \text {. }
$$

If in $\mathfrak{M}$ we replace each $\sigma_{j}$ by $(-1)^{i} s_{j}$, then the new minor $\mathfrak{M}^{\prime}$ has the sign

$$
(-1)^{\left(n-k_{1}+1\right)+\left(n-k_{2}+1\right)+\cdots+\left(n-k_{p+1}+1\right)+1+2+\cdots+p}=(-1)^{N} .
$$

The complementary minor of $\mathfrak{M}$ with elements $a_{i j}$ and $\sigma^{\prime}{ }_{k}$, say $\overline{\mathfrak{M}}$, can be expanded in terms of the $(p+1)$-line minors of the last $p+1$ columns. The term in the expansion of $\overline{\mathfrak{M}}$ corresponding with the minor $\mathfrak{R}$ indicated by the row-indices $q_{1}, q_{2}, \cdots, q_{p+1}$ is provided with the sign

$$
(-1)^{(n+1)+(n+2)+\cdots+(n+p+1)+q_{1}+q_{2}+\cdots+q_{p+1}}=(-1)^{P} .
$$


In $\mathfrak{N}$ again we replace the $\sigma^{\prime}$ by $S^{\prime}$, and the new minor $\mathfrak{N}^{\prime}$ has the sign

$$
(-1)^{\left(n-q_{1}+1\right)+\left(n-q_{2}+1\right)+\cdots+\left(n-q_{p+1}+1\right)+1+2+\cdots+p}=(-1)^{Q} .
$$

Thus the determinant in (9) is the sum of terms each of which is a product of 3 determinants $\mathfrak{M}^{\prime}, \mathfrak{N}^{\prime}, D$ and the factor $(-1)^{M+N+P+Q}$. By simple calculation we see that

$$
M+N+P+Q \equiv(p+1)^{2}(\bmod 2) .
$$

As from our assumption the determinants $D$ are non-negative, it follows that each term in the development of (9) has the positive sign, on account of $(-1)^{(p+1)+(p+1)^{2}}=1$.

The application of Theorem 1 to each $\mathfrak{M}^{\prime}$ and $\mathfrak{N}^{\prime}$ completes the proof.

\section{REFERENCES}

1. H. Brunn, Ein Satz über orthosymmetrische und verwandte Determinanten aus den fundamentalen symmetrischen Funktionen, Zeitschrift für Mathematik und Physik vol. 37 (1892) pp. 291-297.

2. P. C. Rosenbloom, Some properties of absolutely monotonic functions, Bull. Amer. Math. Soc. vol. 52 (1946) p. 460.

3. G. Garbieri, Nuovo teorema algebrico e sua speciale applicazione, Giornale di Mat. vol. 16 (1878) pp. 1-17, or, Sir Thomas Muir, The theory of determinants in the historical order of development, vol. III, pp. 163-165.

4. H. Naegelsbach, Ueber eine Classe symmetrischen Functionen, Sch. Prog., Zweibrücken (1871) pp. 8-11, or Muir, vol. III, pp. 144-148.

UNIVERSITY OF INDONESIA AND

Technische Hogeschool, Delft 